\title{
USO DA CASCA DE COCO VERDE COMO ADSORBENTE NA REMOÇÃO DE METAIS TÓXICOS
}

Francisco W. Sousa e Sarah A. Moreira

Departamento de Engenharia Hidráulica e Ambiental, Universidade Federal do Ceará, Campus do Pici s/n, 60455-760

Fortaleza - CE, Brasil

André G. Oliveira, Rivelino M. Cavalcante e Ronaldo F. Nascimento*

Departamento de Química Analítica e Físico-Química, Universidade Federal do Ceará, Campus do Pici s/n, 60455-760

Fortaleza - CE, Brasil

Morsyleide F. Rosa

Embrapa Agroindústria Tropical-CE, R. Dra. Sara Mesquita, 2270, 60511-110 Fortaleza - CE, Brasil

Recebido em 20/6/06; aceito em 24/1/07; publicado na web em 24/7/07

\begin{abstract}
THE USE OF GREEN COCONUT SHELLS AS ADSORBENTS IN THE REMOVAL OF TOXIC METALS. Green coconut shells were treated with acid, base and hydrogen peroxide solutions for 3, 6, 12 and $24 \mathrm{~h}$ for removing toxic metals from synthetic wastewater. The removal of ions by the adsorbent treated with $0.1 \mathrm{~mol} \mathrm{~L}^{-1} \mathrm{NaOH} / 3 \mathrm{~h}$ was $99.5 \% \mathrm{for}^{2+}$ and $97.9 \%$ for $\mathrm{Cu}^{2+}$. The removal of $\mathrm{Cd}^{2+}, \mathrm{Ni}^{2+}, \mathrm{Zn}^{2+}$, using adsorbent treated with $1.0 \mathrm{~mol} \mathrm{~L}{ }^{-1} \mathrm{NaOH} / 3 \mathrm{~h}$, was $98.5,90.3$ and $95.4 \%$, respectively. Particle size, adsorbent concentration and adsorption kinetics were also studied. An adsorbent size of 60-99 mesh and a concentration of 30-40 g/L for 5 min exposure were satisfactory for maximum uptake of $\mathrm{Pb}^{2+}, \mathrm{Ni}^{2+}, \mathrm{Cd}^{2+}, \mathrm{Zn}^{2+}$ and $\mathrm{Cu}^{2+}$ and can be considered as promising parameters for treatment the aqueous effluents contaminated with toxic metals.
\end{abstract}

Keywords : green coconut shells; toxic metals; wastewater.

\section{INTRODUÇÃO}

O Brasil é um dos maiores produtores mundiais de coco verde e a região Nordeste destaca-se pela produção e consumo, sendo responsável por $75 \%$ da produção nacional ${ }^{1}$. O país produziu uma quantidade de 1,9 bilhões de coco verde em 2004, ficando atrás da Índia, terceiro maior produtor mundial ${ }^{2}$. $\mathrm{O}$ agronegócio do produto no Brasil destaca-se principalmente pelo consumo do líquido do fruto in natura ${ }^{3}$, porém são conhecidas mais de 360 modalidades de aproveitamento industrial ${ }^{1}$. Entretanto, o agroresíduo do fruto imaturo do coco verde tem como um de seus principais problemas ambientais a geração de resíduos sólidos. Estima-se que cerca de 2 milhões de toneladas anuais de cascas são geradas em decorrência do consumo de água de coco verde no Brasil².

Atualmente têm sido realizados esforços para encontrar novas aplicações economicamente viáveis das cascas de coco verde. Neste contexto, a utilização das cascas de coco verde como adsorbente para tratar efluentes contaminados é um campo de atuação viável, devido à abundância e o baixo custo deste material ${ }^{4,5}$.

A literatura relata a utilização de diversos materiais agroindustriais como potenciais adsorbentes na remoção de íons metálicos de águas residuais ${ }^{6-11}$. Entretanto, a maioria dos estudos investiga o processo de preparação de bioadsorbente carbonizado visando o emprego no tratamento de efluentes aquosos contaminados por metais pesados ${ }^{12,13}$. Porém, o processo de reciclagem do material carbonizado é bastante oneroso ${ }^{14}$. Por outro lado, poucos pesquisadores têm se dedicado ao estudo de adsorbentes não carbonizados ${ }^{15}$. Neste contexto, a utilização da casca de coco verde como adsorbente na remoção de metais tóxicos é uma alternativa barata e simples para minimizar os problemas de poluição urbana e ambiental gerados pela disposição destes resíduos, bem como diminuir os custos no tratamento de efluentes provenientes de pequenas indústrias.

*e-mail: ronaldo@ufc.br
O objetivo deste trabalho foi investigar a eficiência de diversos tratamentos químicos dados à casca de coco verde, visando emprego no tratamento de efluentes aquosos contaminados por metais tóxicos. Após o tratamento do material foram estudados os parâmetros de concentração do adsorbente, efeito da granulometria e cinética de adsorção em solução aquosa sintética multielementar de íons metálicos.

\section{PARTE EXPERIMENTAL}

\section{Materiais}

Soluções estoque $1000 \mathrm{mg} / \mathrm{L}$ de íons metálicos foram preparadas a partir de seus respectivos sais $\mathrm{Cu}\left(\mathrm{NO}_{3}\right)_{2} \cdot 6 \mathrm{H}_{2} \mathrm{O}, \mathrm{Zn}\left(\mathrm{NO}_{3}\right)_{2} \cdot 6 \mathrm{H}_{2} \mathrm{O}$, $\mathrm{Cd}\left(\mathrm{NO}_{3}\right)_{2} \cdot 6 \mathrm{H}_{2} \mathrm{O}, \mathrm{Pb}\left(\mathrm{NO}_{3}\right)_{2}$ e $\mathrm{Ni}\left(\mathrm{NO}_{3}\right)_{2} \cdot 6 \mathrm{H}_{2} \mathrm{O}$ de grau analítico Merck (São Paulo, Brasil) utilizando-se água deionizada. A partir da solução estoque foi preparada uma solução padrão multielementar $(100 \mathrm{mg} / \mathrm{L}$ em pH 5,0) para o estudo de adsorção em batelada. Ácido clorídrico $(\mathrm{HCl})$, ácido nítrico $\left(\mathrm{HNO}_{3}\right)$, ácido fosfórico $\left(\mathrm{H}_{3} \mathrm{PO}_{4}\right)$, hidróxido de sódio $(\mathrm{NaOH})$ e peróxido de hidrogênio $\left(\mathrm{H}_{2} \mathrm{O}_{2}\right)$ (Merck, SP, Brasil) foram usados para o estudo do tratamento do adsorbente. Solução tampão de acetato de sódio e ácido acético glacial em pH 5,0 foi utilizada para remoção de traços de $\mathrm{NaOH} 0,1 \mathrm{~mol} \mathrm{~L}^{-1}$. As concentrações dos íons metálicos foram determinadas por espectrofotometria de absorção atômica (EAA) modelo GBC 933 plus.

\section{Obtenção da casca de coco verde}

As cascas de coco verde foram fornecidas pela Embrapa Agroindústria Tropical-CE (EMBRAPA/CE). Inicialmente, as cascas foram dilaceradas (em triturador com facas de corte e martelos desintegradores), em seguida, foram prensadas em prensa "PRH" (prensa de rolos horizontais) e classificadas de forma a se obter duas frações: pó e fibra ${ }^{16}$. Análises de microscopia eletrônica de 
varredura (MEV) foram realizadas para observação das características moforlógicas do material.

\section{Preparação do material adsorbente}

O material obtido foi submetido a um processo de separação granulométrica para obtenção de diferentes frações do resíduo. As frações foram separadas em faixas granulométricas de 60 a 325 mesh $(0,246-0,043 \mathrm{~mm})$ utilizando-se peneiras adequadas (padrão ABNT). Estas frações foram ativadas com soluções ácidas $(\mathrm{HCl}$ 0,1 e $1,0 \mathrm{~mol} \mathrm{~L}^{-1}, \mathrm{HNO}_{3} 0,1$ e $1,0 \mathrm{~mol} \mathrm{~L}^{-1}$ e $_{3} \mathrm{PO}_{4} 0,1$ e $1,0 \mathrm{~mol} \mathrm{~L}^{-1}$ ), soluções básicas ( $\mathrm{NaOH}$ 0,1 e 1,0 mol L-) e peróxido de hidrogênio $\left(\mathrm{H}_{2} \mathrm{O}_{2} 0,1 \mathrm{e} 1,0 \mathrm{~mol} \mathrm{~L}^{-1}\right)$ por 3, 6, 12 e $24 \mathrm{~h}$ à temperatura ambiente. Posteriormente foram lavadas com água destilada, solução tampão (pH 5,0) e colocadas para secar a temperatura ambiente. Em seguida, as frações foram deixadas no dessecador a vácuo até a realização dos experimentos de adsorção.

\section{Capacidade de adsorção do adsorbente}

Os experimentos para estimar a capacidade de adsorção do material foram realizados em duplicata utilizando-se erlenmeyers, contendo massa adequada do adsorbente tratado e não tratado e $10,0 \mathrm{~mL}$ de solução sintética multielementar $\left(\mathrm{Pb}^{+2}, \mathrm{Ni}^{+2}, \mathrm{Cd}^{+2}, \mathrm{Zn}^{+2}\right.$ e $\mathrm{Cu}^{+2}$ ) em uma concentração de 100,0 mg/L, em pH 5,0, mantidos sob agitação à temperatura ambiente durante $24 \mathrm{~h}$. A capacidade de adsorção do adsorbente, $Q_{e}$ (mg do metal/ $\mathrm{g}$ do adsorbente) foi determinada com base na diferença de concentração dos íons metálicos, usando-se a Equação $1^{17}$ :

$$
Q_{e}=\frac{\left(C_{o}-C_{e}\right) V}{m}
$$

onde $C o$ é concentração do soluto na solução inicial (mg/L); $C e$, concentração do soluto no equilíbrio (mg/L); $V$, volume da solução (L); $m$, massa do adsorbente (g).

\section{Estudo do efeito de dosagem}

O efeito da concentração de adsorbente na remoção dos íons metálicos foi verificado para concentrações de adsorbente tratado com $\mathrm{NaOH} 0,1 \mathrm{~mol} \mathrm{~L}^{-1}$ por $3 \mathrm{~h}$ em 10, 20, 30, 40 e $50 \mathrm{~g} / \mathrm{L}$ à temperatura ambiente, solução multielementar de $100 \mathrm{mg} / \mathrm{L}$ e pH 5,0. Foram adicionadas para cada concentração $10 \mathrm{~mL}$ de uma solução multielementar $100 \mathrm{mg} / \mathrm{L}$ e mantidas sob agitação durante 1 h. Em seguida, foram analisadas e determinadas as capacidades de adsorção, de acordo com a Equação $1^{17}$.

\section{Estudo do efeito da granulometria}

Erlenmeyers contendo 2,0 g de cada uma das diferentes frações do adsorbente (60-99; 100-149; 150-199 e 200-325 mesh) e $50 \mathrm{~mL}$ de $\mathrm{NaOH} 0,1 \mathrm{~mol} \mathrm{~L}^{-1}$ foram colocados sob agitação em temperatura ambiente durante $3 \mathrm{~h}$ para aumentar a capacidade de adsorção do material. Após este procedimento, cada uma das diferentes frações do adsorbente foi lavada com água deionizada e solução tampão ( $\mathrm{pH} 5,0)$, para retirar o excesso e traços de $\mathrm{NaOH}$ remanescentes. Em seguida, $0,3 \mathrm{~g}$ de cada uma das diferentes frações do material tratado foi mantida em contato com $10 \mathrm{~mL}$ de uma solução sintética multielementar $\left(\mathrm{Pb}^{+2}, \mathrm{Ni}^{+2}, \mathrm{Cd}^{+2}, \mathrm{Zn}^{+2}\right.$ e $\left.\mathrm{Cu}^{+2}\right)$ em uma concentração de $100 \mathrm{mg} / \mathrm{L}$, em pH 5,0, sob agitação em temperatura ambiente durante $24 \mathrm{~h}$ e analisadas em seguida. Os experimentos foram realizados em duplicata.

\section{Estudo de cinética}

O estudo de cinética foi conduzido em sistema de batelada. Uma série de frascos (erlenmeyer de $125 \mathrm{~mL}$ ) contendo $0,4 \mathrm{~g}$ do adsorbente tratado (60-99 mesh) foi colocada em contato com 10 $\mathrm{mL}$ de solução iônica multielementar $\left(\mathrm{Pb}^{+2}, \mathrm{Ni}^{+2}, \mathrm{Cd}^{+2}, \mathrm{Zn}^{+2}\right.$ e $\left.\mathrm{Cu}^{+2}\right)$ em uma concentração de $100 \mathrm{mg} / \mathrm{L}$, em pH 5,0, sob agitação. Em intervalos pré-determinados foram retiradas alíquotas, filtradas e suas concentrações residuais foram determinadas por espectrofotometria de absorção atômica.

\section{RESULTADOS E DISCUSSÃO}

\section{Seleção do material}

O pó da casca de coco verde foi submetido a diferentes tratamentos químicos com o objetivo de aumentar sua capacidade de adsorção e os resultados obtidos são apresentados na Tabela 1. Podese constatar que os valores de capacidade de adsorção do material submetido aos tratamentos T1 e T2 foram mais expressivos em relação aos outros tratamentos. Também observou-se que o tempo de tratamento do material investigado (3-24 h) não influenciou na sua performance de adsorção.

As percentagens de remoção dos íons metálicos pelo adsorbente

Tabela 1. Capacidade de adsorção dos íons metálicos pelo pó de coco verde com diversos tratamentos químicos

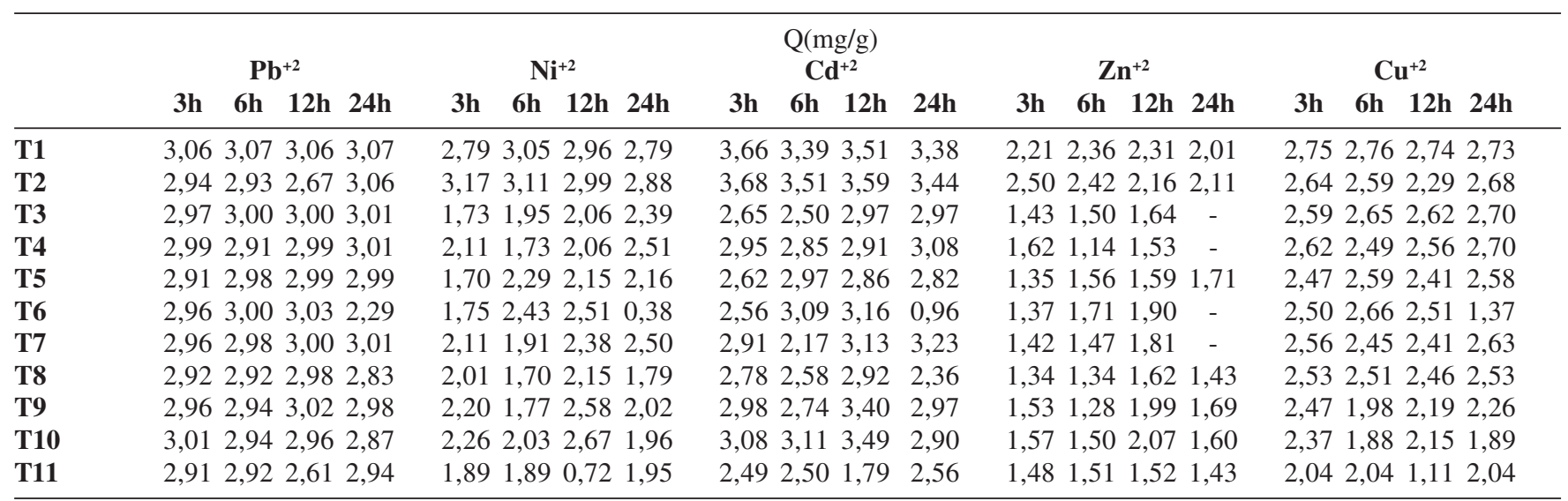

T1 NaOH 0,1 M; T2 NaOH 1,0 M; T3 HCl 0,1 M; T4 HCl 1,0 M; T5 HNO3 0,1 M; T6 HNO3 1,0 M; T7 HzPO 4 0,1 M; T8 $\mathrm{H}_{3} \mathrm{PO}_{4}$ 1,0 M; T9 $\mathrm{H}_{2} \mathrm{O}_{2} 0,1 \mathrm{M} ; \mathrm{T} 10 \mathrm{H}_{2} \mathrm{O}_{2}$ 1,0 M; T11 Bruto; (-) resultados insignificantes. 
tratado e não tratado são mostradas nas Figuras 1-5. As Figuras 1 e 2 mostraram que o pó da casca de coco verde após o tratamento T1 removeu 99,5 e 97,9\% de íons $\mathrm{Pb}^{+2}$ e $\mathrm{Cu}^{+2}$, respectivamente. Entretanto, para os íons $\mathrm{Cd}^{+2}, \mathrm{Ni}^{+2} \mathrm{e} \mathrm{Zn}^{+2}$ remoções de 98,5, 90,3 e 95,4\%, respectivamente, foram obtidas após o tratamento T2 (Figuras 3 a 5). Assim, constatou-se que os tratamentos $\mathrm{T} 1$ e T2 dados ao pó da casca de coco verde apresentaram melhores resultados que o material bruto e os demais tratamentos correspondentes. Entretanto, o tratamento T1 foi escolhido para o desenvolvimento do trabalho, devido à facilidade operacional e por questão de custo. $\mathrm{O}$ tempo selecionado para tratamento do pó da casca de coco verde foi de 3 h, uma vez que não houve uma variação significante na adsorção dos íons metálicos em relação aos demais tempos de tratamentos estudados.

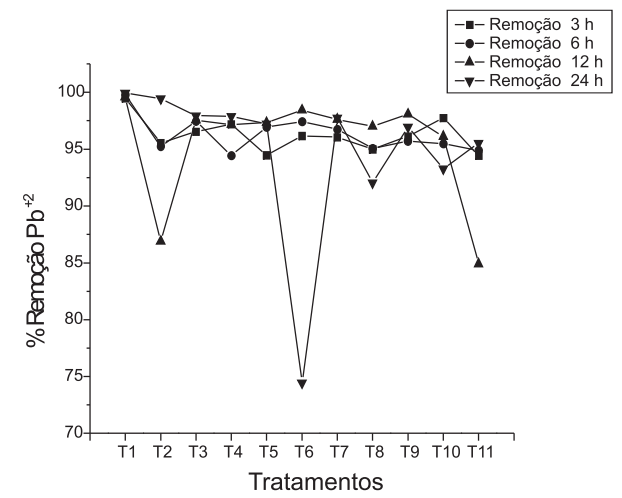

Figura 1. Percentual de remoção do íon $\mathrm{Pb}^{+2}$ na solução multielementar utilizando diferentes tratamentos para os tempos de contato de 3, 6, 12 e $24 \mathrm{~h}$ Tratamentos: T $1 \mathrm{NaOH}$ 0,1 M; T2 NaOH 1,0 M; T3 HCl 0,1 M; T4 HCl 1,0 M; T5 $\mathrm{HNO}_{3} 0,1 \mathrm{M}$; T6 $\mathrm{HNO}_{3} 1,0 \mathrm{M} ; \mathrm{T}_{7} \mathrm{H}_{3} \mathrm{PO}_{4} 0,1 \mathrm{M} ; \mathrm{T}_{8} \mathrm{H}_{3} \mathrm{PO}_{4} 1,0 \mathrm{M} ; \mathrm{T}_{9} \mathrm{H}_{2} \mathrm{O}_{2} 0,1 \mathrm{M} ; \mathrm{T} 10$ $\mathrm{H}_{2} \mathrm{O}_{2} 1,0 \mathrm{M} ; \mathrm{T} 11$ Bruto

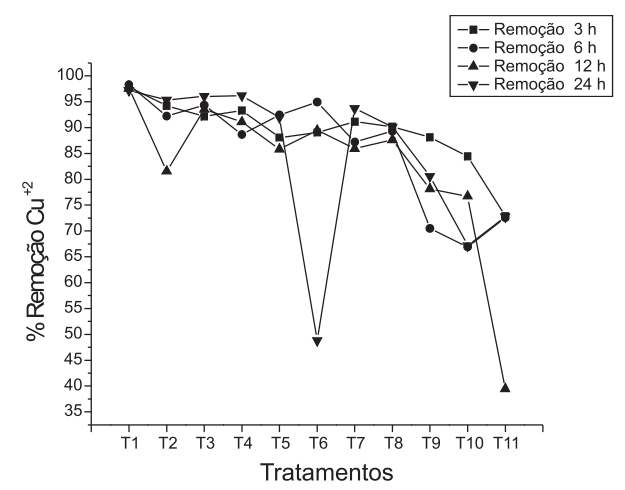

Figura 2. Percentual de remoção do íon $\mathrm{Cu}^{+2}$ na solução multielementar utilizando diferentes tratamentos para os tempos de contato de 3, 6, 12 e $24 \mathrm{~h}$ Condições de tratamento de T1-T11 ver legenda da Figura 1

\section{Estudo do efeito da granulometria}

O efeito da granulometria do adsorbente foi estudado para a faixa de tamanho de partículas de 0,246-0,043 mm (60-325 mesh). Os resultados, apresentados na Figura 6, mostram um aumento similar no percentual de remoção dos íons metálicos para as faixas 60-99, 100-149, 150-199 mesh, enquanto que para a faixa 200-325 mesh observa-se pequena diminuição. Torem e colaboradores ${ }^{4}$, investigando a remoção de íons $\mathrm{Cd}^{+2}$ em casca de coco verde não tratada, também observaram perda da eficiência de remoção com tamanho de partículas de 200-325 mesh.

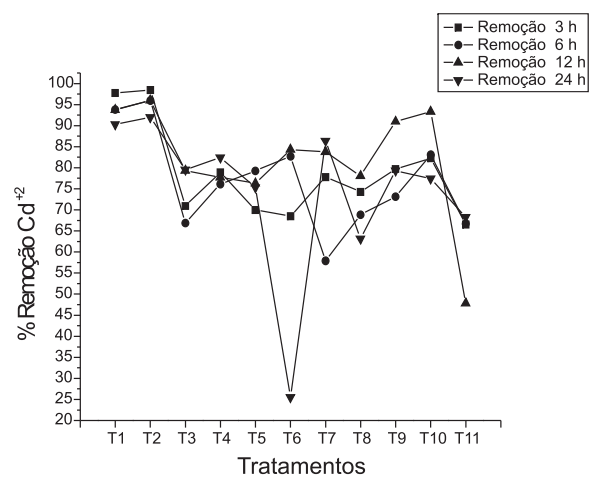

Figura 3. Percentual de remoção do íon $\mathrm{Cd}^{+2}$ na solução multielementar utilizando diferentes tratamentos para os tempos de contato de 3, 6, 12 e $24 \mathrm{~h}$ Condições de tratamento de T1-T11 ver legenda da Figura 1

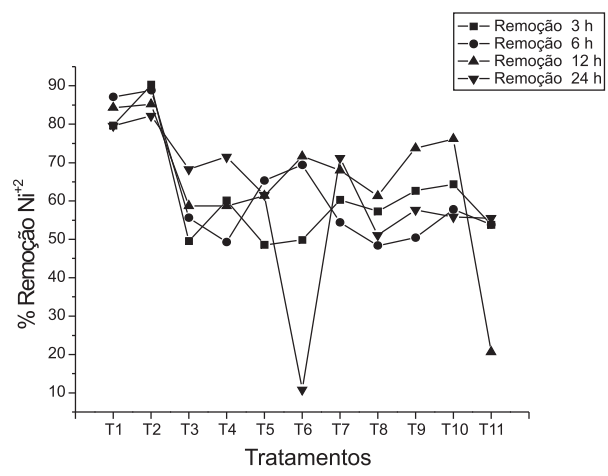

Figura 4. Percentual de remoção do íon $\mathrm{Ni}^{+2}$ na solução multielementar utilizando diferentes tratamentos para os tempos de contato de 3, 6, 12 e $24 \mathrm{~h}$ Condições de tratamento de T1-T11 ver legenda da Figura 1

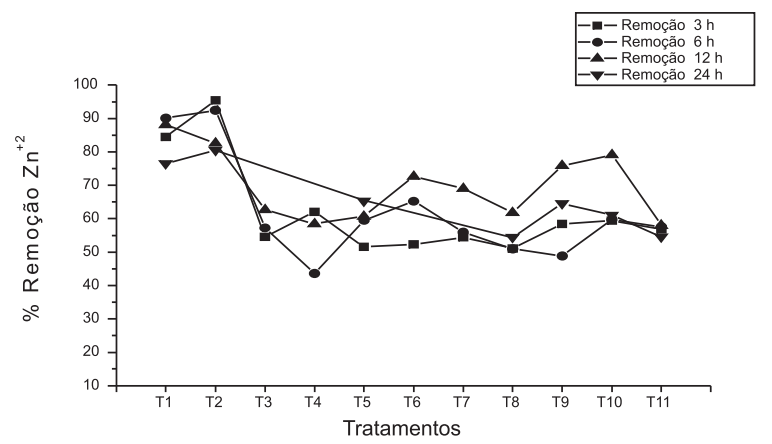

Figura 5. Percentual de remoção do íon $\mathrm{Zn}^{+2}$ na solução multielementar utilizando diferentes tratamentos para os tempos de contato de 3, 6, 12 e $24 \mathrm{~h}$ Condições de tratamento de T1-T11 ver legenda da Figura 1

Uma vez que não houve um significante efeito da granulometria (60-199 mesh) na eficiência de remoção dos íons metálicos, a faixa de 60-99 mesh foi escolhida para o desenvolvimento do trabalho, devido à facilidade de obtenção e operação.

\section{Estudo da superfície do material adsorbente}

O material tratado com $\mathrm{NaOH} 0,1 \mathrm{~mol} \mathrm{~L}^{-1}$ e o material bruto, ambos com granulometria de 60-99 mesh, foram analisados utilizando-se a técnica de microscopia eletrônica de varredura (MEV), com o intuito de verificar as características morfológicas do adsorbente (Figuras 7 e 8).

De modo geral, observa-se que o material é extremamente poroso e apresenta uma superfície bastante irregular. Na Figura 7, pode-se observar que o material bruto é constituído por uma super- 


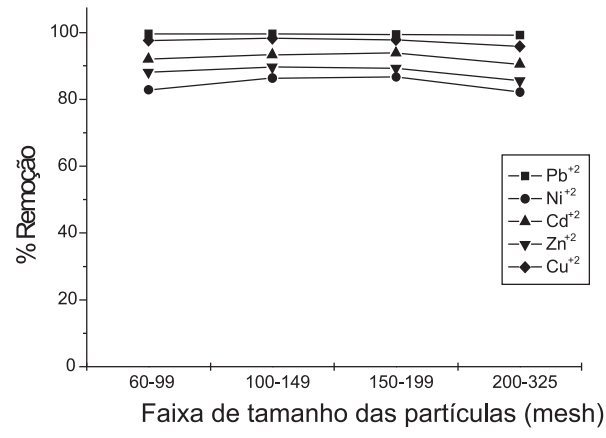

Figura 6. Percentual de remoção dos íons metálicos para diferentes faixas de tamanho de partículas, pH 5,0 e temperatura ambiente

fície porosa com diâmetro dos poros variando de 40-60 $\mu \mathrm{m}$. Na Figura 8 observa-se que a superfície do material tratado é composta de poros com faixa de diâmetro menor que o material bruto, o que favorece a melhor retenção dos íons metálicos.

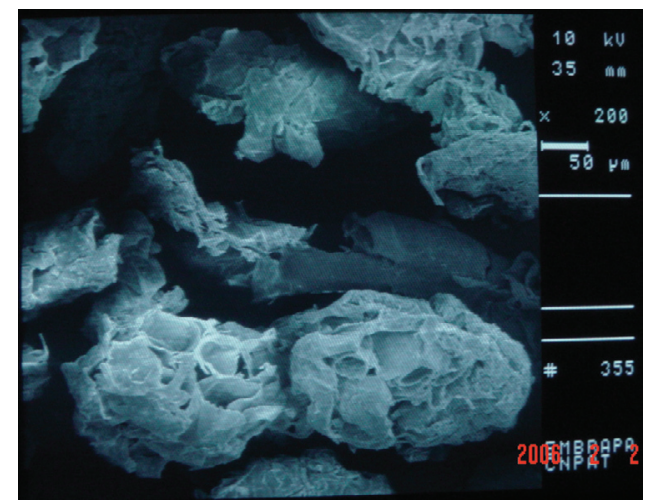

Figura 7. Superficie do material bruto de 60-99 mesh. Diâmetro dos poros igual a 40-60 $\mu \mathrm{m}$

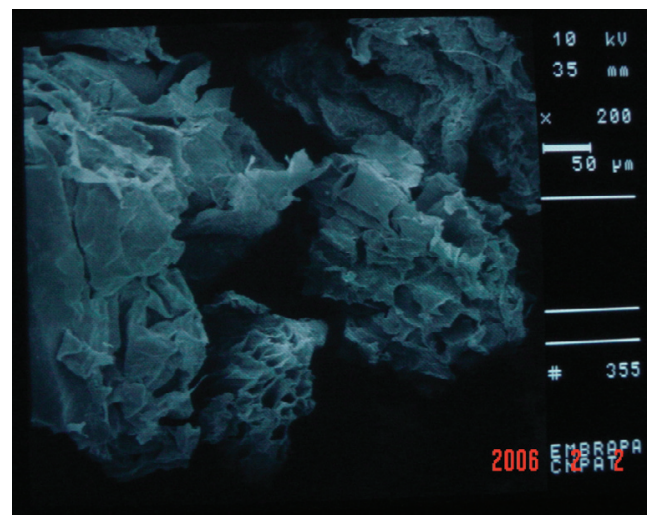

Figura 8. Superfície do material tratado com $\mathrm{NaOH} \mathrm{0,1} \mathrm{mol} \mathrm{L}^{-1}$ de 60-99 mesh. Diâmetro dos poros igual a 40-42 $\mu \mathrm{m}$

\section{Estudo do efeito de dosagem}

Para determinação da quantidade mínima de material adsorbente necessária para a máxima remoção dos íons metálicos, foi realizado o estudo de dosagem com concentrações variando de 10, 20, 30, 40 e $50 \mathrm{~g} / \mathrm{L}$ de adsorbente. Neste estudo, foi encontrado que uma dose de $40 \mathrm{~g} / \mathrm{L}$ do material é suficiente para um máximo de remoção dos íons metálicos $\mathrm{Zn}^{+2}, \mathrm{Ni}^{+2}$, e $\mathrm{Cd}^{+2}$ nas condições mencionadas. Para os metais $\mathrm{Pb}^{+2}$ e $\mathrm{Cu}^{+2}$ a remoção máxima foi verificada com uma concentração de adsorbente de $30 \mathrm{~g} / \mathrm{L}$ (Figura 9).

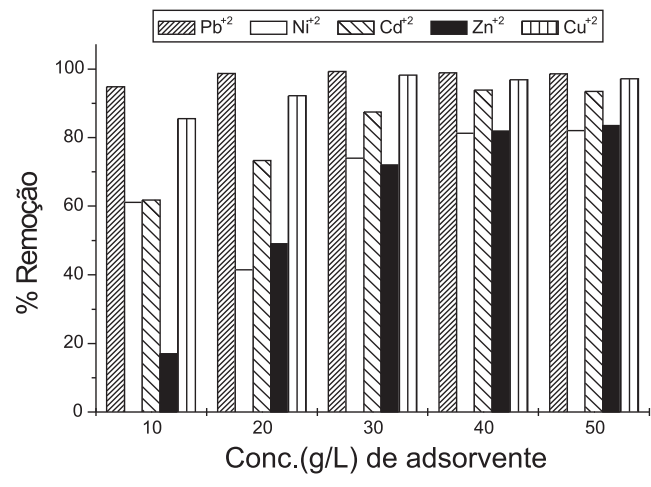

Figura 9. Percentagem de remoção dos íons metálicos em diferentes concentrações de adsorbentes, pH 5,0 e temperatura ambiente

\section{Cinética de adsorção dos íons metálicos}

A cinética de adsorção foi estudada com uma solução multielementar (100 mg/L em pH 5,0) dos íons $\mathrm{Pb}^{+2}, \mathrm{Ni}^{+2}, \mathrm{Cd}^{+2}$, $\mathrm{Zn}^{+2}$ e $\mathrm{Cu}^{+2}$ e o resultado pode ser visto na Figura 10. Observouse que o tempo de equilíbrio de adsorção é bastante rápido, ocorrendo dentro dos primeiros $5 \mathrm{~min}$ para todos os íons metálicos estudados.

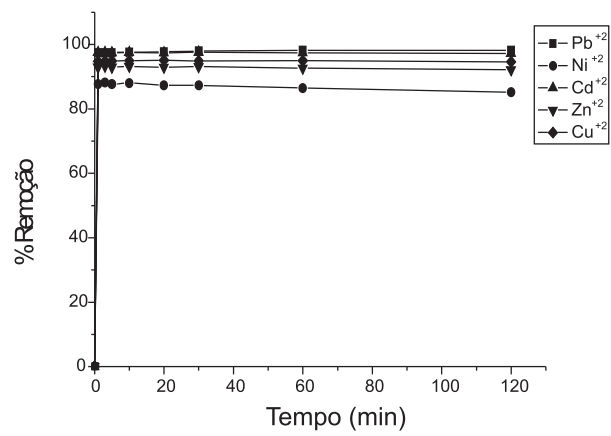

Figura 10. Percentual de remoção dos ions metálicos em solução multielementar (100 mg/L) dos íons $\mathrm{Pb}^{+2}, \mathrm{Ni}^{+2}, \mathrm{Cd}^{+2}, \mathrm{Zn}^{+2}$ e $\mathrm{Cu}^{+2}$, em $\mathrm{pH}$ 5,0 e temperatura ambiente

Os dados experimentais da cinética de adsorção foram analisados usando-se os modelos de cinética de pseudo-primeira ordem, pseudo-segunda ordem e difusão intrapartícula ${ }^{18}$. Para isto foram utilizadas as Equações de Lagergren de primeira ordem (Equação 2) e segunda ordem (Equação 3) e a Equação de difusão intrapartícula (Equação 4).

$$
\begin{aligned}
& \log \left(Q_{e}-Q_{t}\right)=\log Q_{e}-\frac{K_{1}}{2.303} \times t \\
& \frac{t}{Q_{t}}=\frac{1}{K_{2} Q_{e^{2}}}+\frac{1}{Q_{e}} \times t \\
& Q_{t}=K_{f} \times t^{0.5}
\end{aligned}
$$

onde, $Q_{e}$ e $Q_{t}$ são as capacidades de adsorção no equilíbrio e a capacidade individual em um determinado tempo (em $\mathrm{mg} / \mathrm{g}$ ), enquanto que $K_{1}, K_{2}$ e $K_{f}$ são as constantes de primeira ordem, segunda ordem e difusão intrapartícula e $t$ o tempo em min.

A validação dos modelos foi verificada pelo gráfico linear de $\log \left(Q_{e}-Q_{t}\right)$ versus $t$ para a equação de pseudo-primeira ordem, $t /$ $Q_{t}$ versus $t$ para pseudo-segunda ordem e $Q_{t}$ versus $t^{0,5}$ para difusão intrapartícula. As constantes $K_{l}, K_{2}$ e $K_{f}$ mostradas na Tabela 1 foram calculadas através dos coeficientes angulares e lineares das 
Tabela 2. Parâmetros de cinética de adsorção no pó da casca de coco verde tratada com $\mathrm{NaOH} 0,1$ mol L-1 e granulometria de 60-99 mesh

\begin{tabular}{|c|c|c|c|c|c|c|c|c|c|c|}
\hline \multirow[b]{2}{*}{ íon } & \multirow[b]{2}{*}{$\begin{array}{c}\mathrm{C}_{\mathrm{o}} \\
(\mathrm{mg} / \mathrm{L})\end{array}$} & \multirow[b]{2}{*}{$\begin{array}{c}\mathrm{Q}_{\mathrm{e}(\exp )} \\
(\mathrm{mg} / \mathrm{g})\end{array}$} & \multicolumn{3}{|c|}{ Primeira Ordem } & \multicolumn{3}{|c|}{ Segunda Ordem } & \multicolumn{2}{|c|}{ Difusão Intrapartícula } \\
\hline & & & $\begin{array}{c}\mathrm{Q}_{\mathrm{e}(\mathrm{cal})} \\
(\mathrm{mg} / \mathrm{g})\end{array}$ & $\begin{array}{c}\mathrm{K}_{1} \\
\left(\min ^{-1}\right)\end{array}$ & $\mathrm{R}$ & $\begin{array}{c}\mathrm{Q}_{\mathrm{e}(\mathrm{cal})} \\
(\mathrm{mg} / \mathrm{g})\end{array}$ & $\begin{array}{c}\mathrm{K}_{2} \\
(\mathrm{~g} / \mathrm{mg} \min )\end{array}$ & $\mathrm{R}$ & 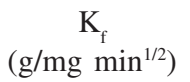 & $\mathrm{R}$ \\
\hline $\mathrm{Pb}^{+2}$ & 79,04 & 1,941 & 1,935 & 0,040 & 0,895 & 1,935 & 23,00 & 1,00 & $2,3 \mathrm{E}-3$ & 0,867 \\
\hline $\mathrm{Ni}^{+2}$ & 80,05 & 1,923 & 1,921 & 0,232 & 0,917 & 1,921 & 41,36 & 1,00 & $3 \mathrm{E}-4$ & 0,024 \\
\hline $\mathrm{Cd}^{+2}$ & 107,0 & 2,654 & 2,654 & 0,034 & 0,984 & 2,654 & 236,4 & 1,00 & $7 \mathrm{E}-5$ & 0,065 \\
\hline $\mathrm{Zn}^{+2}$ & 97,68 & 2,386 & 2,386 & 0,354 & 0,760 & 2,386 & 292,6 & 1,00 & $6 \mathrm{E}-4$ & 0,422 \\
\hline $\mathrm{Cu}^{+2}$ & 85,71 & 2,037 & 2,034 & 0,105 & 0,954 & 2,037 & 54,72 & 1,00 & $2 \mathrm{E}-3$ & 0,984 \\
\hline
\end{tabular}

retas dos gráficos obtidos (não mostrados). Os resultados mostraram que o valore experimental $\left(Q_{e}\right)$ apresenta concordância com o valore calculado $\left(Q_{c}\right)$, para os modelos de pseudo-primeira ordem e pseudo-segunda ordem. Entretanto, a correlação linear de ambos os modelos (R) indica que os resultados de pseudo-segunda ordem estão mais bem correlacionados que o de pseudo-primeira ordem. Estes resultados indicam que a cinética de adsorção do pó da casca de coco verde para uma solução sintética multielementar segue o modelo de pseudo-segunda ordem.

O modelo de difusão intrapartícula foi verificado para o gráfico de $Q_{t}$ versus $t^{0,5}$, o qual preconiza que se o gráfico é linear e passa pela origem o mecanismo de adsorção predominante é o de difusão ${ }^{18}$. Neste estudo, o comportamento do gráfico de $\mathrm{Q}_{t}$ versus t ${ }^{0,5}$ (não mostrado) e o valor do coeficiente de correlação mostraram que não há uma boa correlação linear, nem, uma reta que passe pela origem (Tabela 2).

\section{CONCLUSÕES}

O tratamento básico dado ao pó da casca de coco verde com $\mathrm{NaOH} 0,1 \mathrm{~mol} \mathrm{~L}^{-1} / 3 \mathrm{~h}$ promoveu um aumento na capacidade de adsorção dos metais tóxicos $\mathrm{Pb}^{+2}, \mathrm{Ni}^{+2}, \mathrm{Cd}^{+2}, \mathrm{Zn}^{+2}$ e $\mathrm{Cu}^{+2}$ em solução aquosa sintética multielementar. O estudo do efeito da granulometria mostrou que a performance de adsorção do material não é influenciada para tamanho de partículas variando de 60 a 99 mesh. Uma concentração de adsorvente de $40 \mathrm{~g} / \mathrm{L}$ é suficiente para um máximo de adsorção dos íons metálicos $\mathrm{Zn}^{+2}, \mathrm{Ni}^{+2}$ e $\mathrm{Cd}^{+2}$, enquanto que para os metais $\mathrm{Pb}^{+2}$ e $\mathrm{Cu}^{+2}$ a máxima capacidade de adsorção do adsorvente foi verificada com uma concentração de material de $30 \mathrm{~g} / \mathrm{L}$. O processo de adsorção segue uma cinética de pseudo-segunda ordem. A casca de coco verde, resíduo agroindustrial biodegradável e de fonte renovável, pode ser uma alternativa viável de bioadsorbente de baixo custo para tratamento de efluentes industriais contaminados por metais tóxicos. Baseado nos respectivos resultados, estudos de batelada de adsorção e leito fixo estão em fase de finalização.

\section{AGRADECIMENTOS}

Ao Laboratório de Microscopia Eletrônica e à Embrapa Agroindústria Tropical-CE, pela concessão do aparelho para a realização das micrografias eletrônica por varredura-MEV que viabilizaram a execução deste trabalho.

\section{REFERÊNCIAS}

1. Ferreira Neto, M.; Gheyi, H. R.; Holanda, J. S.; Medeiros, J. F.; Fernandes, P. D.; R. Bras. Eng. Agric. Ambiental 2002, 6, 69.

2. http://secis.mct.gov.br/index.php, acessada em Setembro 2006.

3. Carrijo, O. A.; Vidal, M. C.; Alcântara, C.; Hortic. Bras. 2002, 20, 533.

4. Torem, M. L.; Pino, G. H.; Mesquita, L. M. S.; Pinto, G. A. S.; Miner. Eng. 2006, 19, 380.

5. Sekar, M.; Sakthi, V.; Rengaraj, S.; J. Colloid Interface Sci. 2004, 279, 307.

6. Nasernejad, B.; Zadeh, T. E.; Pour, B. B.; Bygi, M. E.; Zamani, A.; Process Biochem. 2005, 40, 1319.

7. Johnson, P. D.; Watson, M. A.; Brown, J.; Jefcoat, I. A.; Waste Manage. 2002, 22, 471 .

8. Hasan, S. H.; Singh, K. K.; Rastogi, R.; J. Hazard. Mater. 2005, A121, 51.

9. Kim, D. S.; Kim, J. W.; Sohn, M. H.; Sohn, S. M.; Kwon, Y. S.; J. Hazard. Mater. 2001, B85, 301

10. Petroni, S. L.; Pires, M. A. F.; Munita, C. S.; Quim. Nova 2000, 23, 477.

11. Lamim, A. P. B.; Jordão, C. P.; Pereira, J. L.; Bellato, C. R.; Quim. Nova 2001, 24, 18 .

12. Ho, Y.S.; Ofomaja, A.; Biochem. Eng. J. 2006, 30,117.

13. Kadirvelu, K.; Namasivayam, C.; Environ. Res. 2003, 7, 471.

14. Agarwal, G.S.; Bhuptawat, H. K.; Chaudhari, S.; Bioresour. Technol. 2006, 97, 949.

15. Farajzadeh, M. A.; Monji, A. B.; Sep. Purif. Technol. 2004, 38, 97.

16. Rosa, M. F.; Figueiredo, M. C. B.; Mattos, A. L. A.; Bezerra, F. C.; Crisostómo, L. A.; Araújo, A. M.; Abreu, F. A. P.; Veras, L. G. C.; Silva, J. T.; Resúmenes de la $50^{a}$ Reunión de la Sociedad Interamericana de Horticultura Tropical, La Mercedes de Guácimo, Costa Rica, 2004.

17. Ruthven, M. D.; Principles of adsorption and adsorption processes, Jonh Wiley \& Sons: New York, 1984.

18. Ho, Y. S.; McKay, G.; Water Res. 1999, 33, 578. 